\title{
Arrays of magnetic nanoindentations with perpendicular anisotropy
}

\author{
D. Makarov, L. Baraban, I. L. Guhr, and J. Boneberg \\ Department of Physics, University of Konstanz, D-78457 Konstanz, Germany \\ H. Schift and J. Gobrecht \\ Laboratory for Micro- and Nanotechnology, Paul Scherrer Institute, CH-5232 Villigen, Switzerland
}

G. Schatz, P. Leiderer, and M. Albrecht ${ }^{\mathrm{a})}$

Department of Physics, University of Konstanz, D-78457 Konstanz, Germany

(Received 7 December 2006; accepted 21 January 2007; published online 1 March 2007)

\begin{abstract}
The authors report an approach to the fabrication of periodic magnetic patterns using spherical indentations. These troughs are shaped by an imprint technique, employing a self-assembled monolayer of particles. Well-defined structures with perpendicular magnetic anisotropy are obtained by deposition of $\mathrm{Co} / \mathrm{Pd}$ multilayer films onto the topographic array. The structures formed in the indentations can be magnetically exchange decoupled from their neighbors depending critically on their specific shape. The presented concept leads to a realization of a magnetic nanostructure medium, which offers advances in achieving high pattern densities combining a low cost nanoimprint approach with material functionalization. (C) 2007 American Institute of Physics.
\end{abstract}

[DOI: 10.1063/1.2709513]

The development of advanced lithography tools to fabricate magnetic structures controllably at the nanoscale has resulted in an enormous growth of studies concerning the physical understanding of nanomagnetism, such as coupling phenomena and scaling behavior, but also future applications, where magnetic nanostructure arrays are envisioned as a magnetic storage medium. ${ }^{1}$ In conventional lithography a pattern is typically created in a resist layer which is subsequently transferred to a magnetic film. The pattern generation can be accomplished with a number of technologies such as electron beam lithography, ${ }^{2,3}$ ion beam lithography, ${ }^{4-8}$ near field lithography, ${ }^{9,10}$ laser interference lithography, ${ }^{11-14}$ x-ray lithography, ${ }^{15,16}$ or nanoimprint. ${ }^{17,18}$ Unfortunately, most methods may not be suitable for largescale device fabrication because they are multistep, expensive, and involve time-consuming procedures. Alternative ways to produce a template for patterning are self-assembly techniques such as anodized alumina, ${ }^{19,20}$ phase separation in block copolymers ${ }^{21-23}$ surface instability induced by ion bombardment, ${ }^{24,25}$ or self-assembled particles. ${ }^{26-28}$ These templates can be used as an etch mask or a deposition mask. Moreover, self-assembled particle monolayers can also be directly employed for nanostructuring, for instance, by depositing multilayered $\mathrm{Co} / \mathrm{Pd}$ films onto the surface of polystyrene spheres. In this regard, the formation of magnetic single domain $50 \mathrm{~nm}$ island caps with perpendicular magnetic anisotropy has been demonstrated. ${ }^{29,30}$

Here we present an analogous approach to the fabrication of periodic patterns of magnetic structures using spherical indentations followed by $\mathrm{Co} / \mathrm{Pd}$ film deposition. These indentations are shaped by an imprint approach employing a self-assembled monolayer of spherical particles.

For this purpose, densely packed two-dimensional arrays of monodisperse polystyrene particles with fixed diameters ranging from 130 to $410 \mathrm{~nm}$ are formed via self-assembling upon slow evaporation of a solvent under ambient conditions

\footnotetext{
a) Author to whom correspondence should be addressed. Electronic mail:
} manfred.albrecht@uni-konstanz.de on a glass substrate. ${ }^{31}$ These particle arrays were covered by a liquid mixture of the prepolymer polydimethylsiloxane (PDMS) and a curing agent (ratio 10:1), as illustrated in Fig. 1. After thermal curing of the sample at $65{ }^{\circ} \mathrm{C}$ for $24 \mathrm{~h}$ polystyrene particles were detached from the hardened surface of the PDMS similar to an approach reported by Nam et al. ${ }^{32} \mathrm{~A}$ scanning electron microscopy (SEM) and an atomic force microscopy (AFM) image of the resulting mold are presented in Fig. 2, revealing well-defined spherical segment indentations, and for demonstration purposes, also areas where particles from the mask remained after mask removal. Note that these defects can be simply avoided by properly dissolving the polystyrene (PS) particles. ${ }^{32}$ Alternatively, such a topographic pattern was also fabricated by electrochemical deposition of a 1-mm-thick Ni film onto the particle assembly followed by the removal of the particle monolayer, as shown in Fig. 1(b). This Ni mask can be directly used for film deposition or as a master to form a stamp in a soft mold, which in turn can be used for imprinting.

The topographic pattern, in particular, the specific shape of the indentation, was investigated by AFM. Depending on



FIG. 1. Schematic drawings showing the various steps for fabricating magnetic arrays of spherical indentations. 


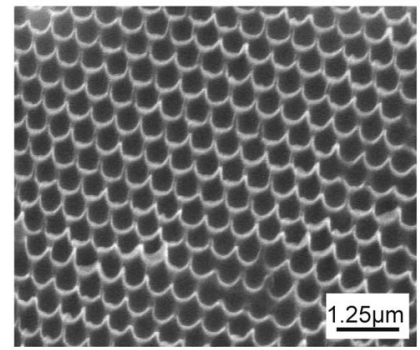

(b) AFM

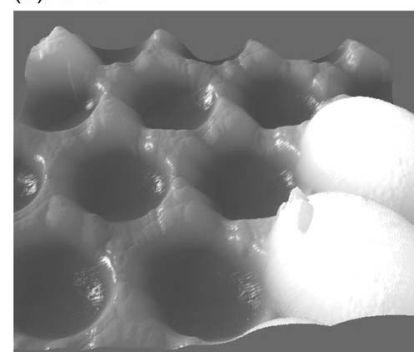

FIG. 2. (a) SEM and (b) AFM images of the mold, revealing well-defined spherical indentations. In addition, a defect area where particles from the mask remained after mask removal is presented for demonstration purposes.

the preparation conditions during the imprint process (viscosity and adhesive property of the PDMS) different shapes can be achieved in the mold, ${ }^{32}$ as exemplified in Fig. 3(a). The shape can be expressed by the ratio between the width $W$ and depth $D$ of the spherical indentation. Note that the filling factor will also be reduced with decreasing width $W$ of the indentions for a fixed particle diameter $d$. The optimal shape concerning magnetic exchange isolation of the deposited $\mathrm{Co} / \mathrm{Pd}$ multilayered film is expected if the spheres of the stamp are embedded into the PDMS film with half of their diameter. In this case the $D / W$ value is equal to 0.5 . Experimental $D / W$ values were extracted from AFM line scans and are summarized in Table I as a function of the employed particle diameter $d$. The results show strong deviations from indentations formed by hard spheres $\left(D / W_{\mathrm{HS}}\right)$, which are most likely due to elastic and viscoelastic relaxations of the indentation after the thermal curing process in PDMS. ${ }^{33}$

(a)

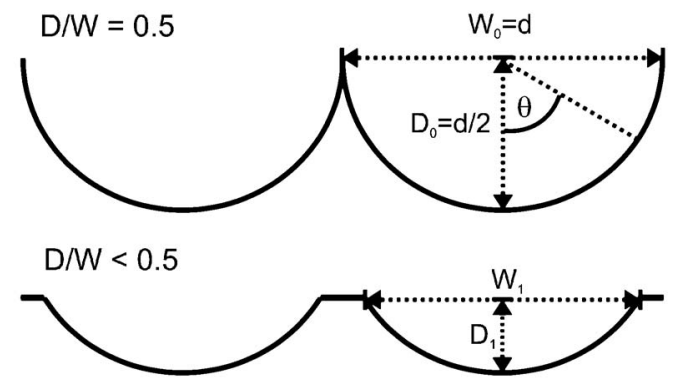

(b)

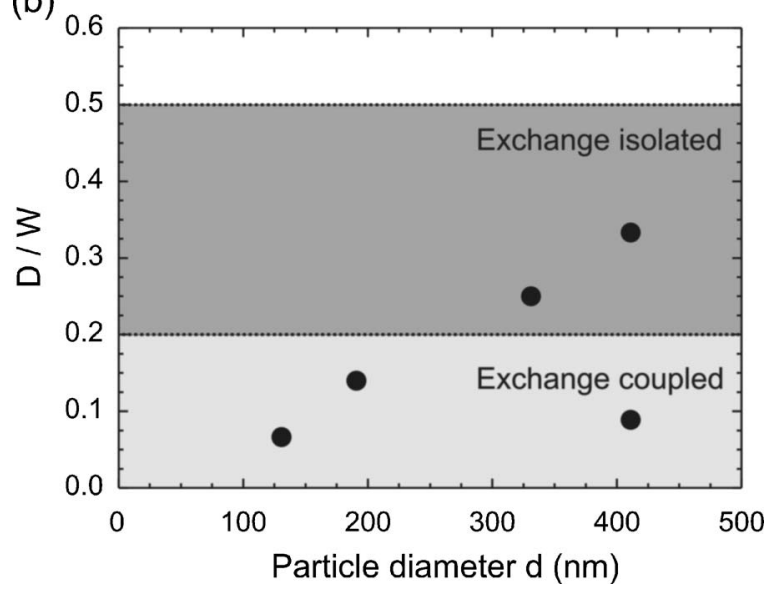

FIG. 3. (a) Shape of the spherical indentation as a function of particle penetration. (b) $D / W$ ratio (full dots) of the fabricated magnetic arrays using various particle sizes. Exchange isolation of the formed magnetic structures is achieved for $0.2<D / W \leqslant 0.5$.
TABLE I. Geometry of the shape of the formed nanoindentations.

\begin{tabular}{ccccc}
\hline \hline $\begin{array}{c}\text { Particle } \\
\text { diameter } \\
d(\mathrm{~nm})\end{array}$ & $\begin{array}{c}\text { Indentation } \\
\text { width } \\
W(\mathrm{~nm})\end{array}$ & $\begin{array}{c}\text { Indentation } \\
\text { depth } \\
D(\mathrm{~nm})\end{array}$ & $\begin{array}{c}D / W \\
\left(D / W_{\mathrm{HS}}\right)\end{array}$ & $\begin{array}{c}\text { Filling } \\
\text { factor }\end{array}$ \\
\hline 130 & 60 & 4 & $0.07(0.12)$ & 0.19 \\
190 & 100 & 14 & $0.14(0.15)$ & 0.25 \\
330 & 220 & 55 & $0.25(0.22)$ & 0.40 \\
410 & 300 & 100 & $0.33(0.24)$ & 0.49 \\
410 & 290 & 25 & $0.09(0.24)$ & 0.48 \\
\hline \hline
\end{tabular}

As already mentioned these patterns act as a topographic pattern and are combined with magnetic film deposition [see Fig. 1(d)]. The material deposition was carried out in a molecular beam epitaxy chamber, operating at a base pressure of $2 \times 10^{-10}$ mbar. $\mathrm{Co} / \mathrm{Pd}$ multilayer films were deposited at room temperature on the prepared patterns by evaporation of $\mathrm{Co}$ and $\mathrm{Pd}$ from two e-beam sources. In our studies a multilayer consisting of a $[\mathrm{Co}(0.3 \mathrm{~nm}) / \mathrm{Pd}(0.8 \mathrm{~nm})]_{12}$ stack deposited on a 5-nm-thick Pd seed layer was used and covered by an additional $0.8-\mathrm{nm}$-thick Pd layer to prevent oxidation. Continuous films grown under the same condition on a sapphire substrate have revealed a perpendicular magnetic anisotropy $\left(K_{u}=0.4 \mathrm{MJ} / \mathrm{m}^{3}\right)$ with a coercivity of about $75 \mathrm{mT}^{29,34}$ The perpendicular magnetic anisotropy originates primarily from interface contributions due to the asymmetry of Co and Pd layers perpendicular to the interface. ${ }^{34}$ Thus, it is assumed that the anisotropy axis will always point perpendicular to the surface of the indentation.

$\mathrm{Co} / \mathrm{Pd}$ film deposition onto the patterns can result in exchange-isolated magnetic structures formed in the indentations which are separated by a network of magnetic material surrounding the array of indentations. Whether magnetic exchange isolation is actually achieved depends on the specific shape and curvature of the indentations, which is expressed by the $D / W$ value. Assuming an evaporation direction perpendicular to the substrate plane, the spherical shape will lead to a reduction in $\mathrm{Co}$ and $\mathrm{Pd}$ thickness along the inner surface of the indentation. Since a typical Co layer thickness of $0.3 \mathrm{~nm}$ corresponds to only about $1.5 \mathrm{ML}$, we expect the ferromagnetic properties of the multilayer stack to be suppressed for a critical angle $\theta$ higher than about $50^{\circ}$, where the Co layer thickness drops below $1 \mathrm{ML} .^{30}$ This critical angle corresponds to a $D / W$ value equal to 0.2 , as exemplified in Fig. 3(a). Thus, indentations with $D / W$ values greater than 0.2 will provide exchange isolation while more shallow arrays with $D / W<0.2$ are expected to be fully exchange coupled, as marked in Fig. 3(b).

The magnetic properties of the samples were investigated by magnetic force microscopy (MFM). Two patterns were formed with the largest particle size of $410 \mathrm{~nm}$ revealing two different $D / W$ values of 0.3 and 0.09 . Figure 4 shows MFM images which have been taken in the remanent state after exposing the samples to a reverse magnetic field perpendicular to the substrate close to the coercivity field. While the pattern with shallow indentations reveals a rather exchange coupled magnetic pattern, the pattern with $D / W$ $=0.3$ shows a uniform white or dark magnetic contrast on each indentation, which suggests that the magnetic structures are in an isolated single domain state with the averaged magnetization pointing perpendicular to the substrate surface, as expected for an array with $D / W>0.2$. To measure the 


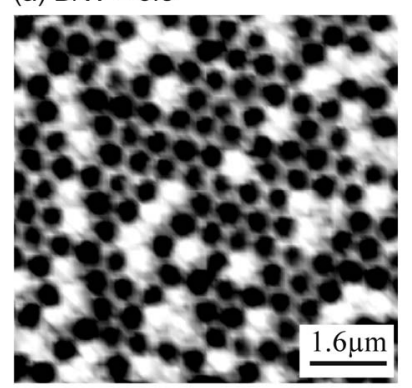

(b) $\mathrm{D} / \mathrm{W}=0.09$

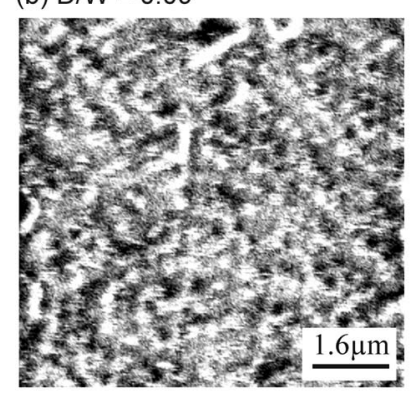

FIG. 4. (a) MFM image of an array with $D / W=0.3$ fabricated by employing $410 \mathrm{~nm}$ particles. (b) MFM image of a magnetically exchange coupled array with $D / W=0.09$.

switching behavior of the exchange-isolated array, MFM images in the remanent state have been analyzed. A remanence curve has been constructed by counting the number of reversed versus unreversed structures as a function of reverse field applied perpendicular to the substrate. This makes the measurement only semiquantitative, as we are not able to accurately measure magnetization with the MFM. However, an averaged switching field of about $100 \mathrm{mT}$ with a rather broad switching field distribution of about $30 \mathrm{mT}$ was found. An enhancement in coercivity and an increase in switching field distribution have been previously observed in patterned systems and have been mainly attributed to the reduced amount of effective nucleation centers for the single domain structures and to a change in the magnetization reversal mechanism in comparison with the continuous ${ }^{35}$ film. ${ }^{30}$ However, due to the magnetic material surrounding the pattern and the different magnetic configurations of the pattern array, magnetostatic coupling will ultimately limit the switching field distribution. Note that for patterns formed with the smallest particle size of $130 \mathrm{~nm}, D / W$ values greater than 0.2 could not be achieved (see Table I).

In conclusion, we have presented an approach to fabricate periodic patterns of magnetic structures, using an imprint technique which is based on self-assembled particle arrays. Well-defined structures with perpendicular magnetic anisotropy are obtained by deposition of $\mathrm{Co} / \mathrm{Pd}$ multilayer films onto the topographic array. The magnetic structures formed in the indentations can be magnetically exchange decoupled from their neighbors, depending on their specific shape as given by their $D / W$ ratio. In addition, further magnetic functionality can be achieved by changing the specific layer stack of the deposited film material where its thickness depends strongly on the curvature of the indentations, determining a spatial gradient in the material properties. However, elastic and viscoelastic relaxations of the spherical indentation after the thermal curing process in PDMS limit this approach, hence metallic masters formed by electrochemical deposition seem to be more promising in order to achieve magnetic exchange-isolated nanoscale patterns. The following low cost imprint process might be more suitable for applications than magnetic patterns formed directly on nanoparticle arrays which might be applied to various fields such as spintronic elements or biomedical engineering.
The authors thank K. Vogelsang (PSI) for performing imprint experiments and R. Bischofberger (AMS GmbH) for the

$\mathrm{Ni}$ galvanic coating. Financial support by the Deutsche Forschungsgemeinschaft through the SFB 513 and the Emmy-Noether program is gratefully acknowledged.

${ }^{1}$ T. Thomson and B. D. Terris, J. Phys. D 38, R199 (2005).

${ }^{2}$ C. A. Ross, Annu. Rev. Mater. Res. 31, 203 (2001).

${ }^{3}$ J. C. Lodder, J. Magn. Magn. Mater. 272-276, 1692 (2004).

${ }^{4}$ J. G. Zhu, X. D. Lin, L. J. Guan, and W. Messner, IEEE Trans. Magn. 36, 23 (2002).

${ }^{5}$ C. T. Rettner, S. Anders, T. Thomson, M. Albrecht, Y. Ikeda, M. E. Best, and B. D. Terris, IEEE Trans. Magn. 38, 1725 (2002).

${ }^{6}$ C. T. Rettner, S. Anders, J. E. E. Baglin, T. Thomson, and B. D. Terris, Appl. Phys. Lett. 80, 279 (2002).

${ }^{7}$ R. Hyndman, P. Warin, J. Gierak, J. Ferre, J. N. Chapman, J. P. Jamet, V. Mathet, and C. Chappert, J. Appl. Phys. 90, 3843 (2001).

${ }^{8}$ B. D. Terris, L. Folks, D. Weller, J. E. E. Baglin, A. J. Kellock, H. Rothuizen, and P. Vettiger, Appl. Phys. Lett. 75, 403 (1999).

${ }^{9}$ M. Naya, I. Tsuruma, T. Tani, and A. Mukai, Appl. Phys. Lett. 86, 201113 (2005).

${ }^{10}$ T. Ito, T. Yamada, Y. Inao, T. Yamaguchi, N. Mizutani, and R. Kuroda, Appl. Phys. Lett. 89, 033113 (2006).

${ }^{11}$ A. Fernandez, P. J. Bedrossian, S. L. Baker, S. P. Vernon, and D. R. Kania, IEEE Trans. Magn. 32, 4472 (1996).

${ }^{12}$ Y. Hao, F. J. Castano, C. A. Ross, B. Vogeli, M. E. Walsh, and H. I. Smith, J. Appl. Phys. 91, 7989 (2002).

${ }^{13}$ S. Sindhu, M. A. M. Haast, K. Ramstock, L. Abelmann, and J. C. Lodder, J. Magn. Magn. Mater. 238, 246 (2002).

${ }^{14}$ A. Carl, S. Kirsch, J. Lohau, H. Weinforth, and E. F. Wassermann, IEEE Trans. Magn. 35, 3106 (1999).

${ }^{15}$ H. I. Smith, J. Vac. Sci. Technol. B 13, 2323 (1995).

${ }^{16} \mathrm{C}$. Miramond, C. Fermon, F. Rousseaux, D. Decanini, and F. Carcenac, J. Magn. Magn. Mater. 165, 500 (1997).

${ }^{17}$ S. Y. Chou, Proc. IEEE 85, 652 (1997).

${ }^{18}$ G. M. McClelland, M. W. Hart, C. T. Rettner, M. E. Best, K. R. Carter, and B. D. Terris, Appl. Phys. Lett. 81, 1483 (2002).

${ }^{19}$ P. Aranda and J. M. Garcia, J. Magn. Magn. Mater. 249, 214 (2002).

${ }^{20}$ H. Masuda, H. Yamada, M. Satoh, H. Asoh, M. Nakao, and T. Tamamura, Appl. Phys. Lett. 71, 2770 (1997).

${ }^{21}$ C. Harrison, D. H. Adamson, Z. D. Cheng, J. M. Sebastian, S. Sethuraman, D. A. Huse, R. A. Register, and P. M. Chaikin, Science 290, 1558 (2000).

${ }^{22}$ C. Park, J. Y. Cheng, M. J. Fasolka, A. M. Mayes, C. A. Ross, E. L. Thomas, and C. De Rosa, Appl. Phys. Lett. 79, 848 (2001).

${ }^{23}$ J. Y. Cheng, C. A. Ross, E. L. Thomas, H. I. Smith, and G. J. Vancso, Appl. Phys. Lett. 81, 3657 (2002).

${ }^{24}$ S. Facsko, T. Dekorsy, C. Koerdt, C. Trappe, H. Kurz, A. Vogt, and H. L. Hartnagel, Science 285, 1551 (1999).

${ }^{25}$ Y. J. Chen, J. P. Wang, E. W. Soo, L. Wu, and T. C. Chong, J. Appl. Phys. 91, 7323 (2002)

${ }^{26}$ C. L. Cheung, R. J. Nikolić, C. E. Reinhardt, and T. F. Wang, Nanotechnology 17, 1339 (2006).

${ }^{27}$ P. W. Nutter, H. Du, V. Vorithitikul, D. Edmundson, E. W. Hill, J. J. Miles, and C. D. Wright, IEEE Proc.-Sci. Meas. Technol. 150, 227 (2003).

${ }^{28}$ M. Zhu, Y. Li, T. Meng, P. Zhan, J. Sun, J. Wu, Z. Wang, S. Zhu, and N. Ming, Langmuir 22, 7248 (2006).

${ }^{29}$ M. Albrecht, G. Hu, I. L. Guhr, T. C. Ulbrich, J. Boneberg, P. Leiderer, and G. Schatz, Nat. Mater. 4, 203 (2005).

${ }^{30}$ T. C. Ulbrich, D. Makarov, G. Hu, I. L. Guhr, D. Suess, T. Schrefl, and M. Albrecht, Phys. Rev. Lett. 96, 077202 (2006).

${ }^{31}$ U. C. Fischer and H. P. Zingsheim, J. Vac. Sci. Technol. 19, 881 (1981).

${ }^{32}$ H. J. Nam, D.-Y. Jung, G.-R. Yi, and H. Choi, Langmuir 22, 7358 (2006).

${ }^{33}$ Y.-T. Cheng, W. Ni, and C.-M. Cheng, Phys. Rev. Lett. 97, 075506 (2006).

${ }^{34}$ M. T. Johnson, P. J. H. Bloemen, F. J. A. den Broeder, and J. J. de Vries, Rep. Prog. Phys. 59, 1409 (1996).

${ }^{35}$ T. Thomson, G. Hu, and B. D. Terris, Phys. Rev. Lett. 96, 257204 (2006). 